\title{
Clinical AND EPIDEMIOLOGICAL PROFILE OF HCV GENOTYPE 3 PATIENTS IN SOUTHERN BRAZIL
}

\author{
Marisa Boff Costa ${ }^{1}$, Larisse Longo ${ }^{1}$, Deivid Santos ${ }^{2}$, Raquel Boff da Costa ${ }^{1}$, \\ Gustavo Hirata Dellavia ${ }^{2}$, Soraia Arruda ${ }^{3}$, Matheus Truccolo Michalczuk ${ }^{1,3}$, \\ Mário Reis Álvares-da-Silva ${ }^{1,3}$
}

\begin{abstract}
Introduction: Despite the emergence of new treatments for HCV genotype 3 (HCV G3), there is still a lack of data about this particular subgroup in Brazil. Our objective was to describe clinical and sociodemographic variables and treatment profile of HCV G3 Brazilian patients.
\end{abstract}

Methods: This was a descriptive, retrospective study, performed in a specialized center for HCV treatment in the South Region of Brazil. Medical records of patients diagnosed with HCV G3 were reviewed to collect clinical, sociodemographic, and treatment information.

Results: Participants included total of 564 patients, with a mean age of 59.3 years $(S D=10.5)$. Cirrhosis was present in $54.4 \%$ of patients. The most common coexisting conditions were systemic arterial hypertension (36.6\%) and diabetes mellitus $(30 \%)$. Regarding treatment, $25.2 \%$ of the patients were treatment-naïve and $74.8 \%$ were currently under treatment $(11.6 \%)$ or had received a previous treatment $(87 \%)$. The most frequent ongoing treatment was sofosbuvir + daclatasvir ( \pm ribavirin) $(87.8 \%)$. Of the 388 patients who had at least one previous treatment, $67 \%$ achieved sustained virologic response in the last treatment. Caucasian / white, non-obese, transplanted patients, those with longer time since diagnosis and with cirrhosis were more likely to receive treatment, according to multivariate analysis. Patients with hepatocellular carcinoma were $64.1 \%$ less likely to be on treatment during the study period than those without this condition; patients with chronic kidney disease were 2.91-fold more likely to have an interruption of treatment than those without this condition.

Conclusion: This study describes a large sample of Brazilian patients with HCV G3. Treatment patterns were mainly influenced by the presence of HCV complications and comorbidities.

Keywords: Hepatitis C; liver cirrhosis; treatment; HCV-3; genotypes

According to the World Health Organization, hepatitis C virus (HCV) infection affects approximately $1 \%$ of global population, being the underlying cause of 1.34 deaths in $2015^{1}$. In Brazil, it is estimated that $0.7 \%$ of the population has been exposed to the virus and 657,000 of them are effectively viraemic, requiring treatment according to data from $2016^{2}$.

$\mathrm{HCV}$ presents a broad genetic diversity, which poses a challenge to the management of the disease. In total, there are seven genotypes and at least 67 subtypes $^{3}$. Genotypes 1 and 3 are the most prevalent worldwide, present in $44 \%$ and $25 \%$ of HCV cases, respectively ${ }^{4}$. In Brazil, a similar pattern is observed, however, $64.9 \%$ of HCV patients have genotype 1 , and $30.2 \%$ of HCV patients have genotype 3 . Also, as a continental country, the distribution of Brazilian genotypes may vary, but always with genotype 1 as the most prevalent ${ }^{5}$. Comparatively to genotype 1 , genotype 3 is associated with a significant increase in the risk of cirrhosis and hepatocellular carcinoma ${ }^{6}$. This genotype is also considered easy to treat due to its higher rates of sustained virologic response ${ }^{7}$.

Clin Biomed Res. 2020;40(1):7-13

1 Programa de Pós-graduação em Gastroenterologia e Hepatologia, Universidade Federal do Rio Grande do Sul (UFRGS). Porto Alegre, RS, Brasil.

2 Faculdade de Medicina, Universidade Federal do Rio Grande do Sul (UFRGS). Porto Alegre, RS, Brasil.

3 Serviço de Gastroenterologia, Hospital de Clínicas de Porto Alegre (HCPA). Porto Alegre, RS, Brasil.

Corresponding author: Marisa Boff Costa maricosta@hcpa.edu.br Hospital de Clínicas de Porto Alegre (HCPA) Rua Ramiro Barcelos, 2350 90035-903, Porto Alegre, RS, Brasil. 
$\mathrm{HCV}$ medications, as a high-cost treatment, are mainly provided to patients by the Brazilian federal government. The prescription and use of these drugs follow strict rules described in a Directive of the Ministry of Health. Between 2011 and 2018, new updates of this protocol were published, driven by the recent emergence of new HCV therapies with proven clinical benefit ${ }^{2,8-10}$.

Despite recent advances in disease management, there is a lack of data exclusively about genotype 3 that allows for planning patient care and allocating resources within the healthcare system. South Brazil is the geographic region with highest prevalence of this genotype. Thus, the aims of this study are: (i) to describe clinical and sociodemographic profile of patients with HCV genotype 3; (ii) to describe the treatment profile of these patients and (iii) to evaluate the associations between treatment patterns and clinical characteristics of patients in the southernmost region of Brazil attending a specialized center.

\section{METHODS}

This is was a descriptive, retrospective study, performed in a specialized center for HCV treatment in the South Region of Brazil. Eligible patients were those with confirmed diagnosis of HCV genotype 3 according to medical records who attended at least one outpatient visit between January 2011 and December 2016, aged at least 18 years old, regardless of their classification of fibrosis severity. No restrictions were applied to comorbidities or co-infections. Patients with mixed genotype were excluded. Eligible patients had their medical records reviewed to collect clinical, sociodemographic, and disease characteristics and information on the treatment of HCV and co-infections. This study was approved by the Research Ethics Committee of the Hospital de Clínicas de Porto Alegre under approval number CAAE 67894217.5.0000.5327 and followed the recommended guidelines for studies in humans.

\section{Statistical analysis and sample size calculation}

An exploratory analysis was performed using tabulation measures of central tendency (mean) and dispersion (standard deviation-SD) for quantitative variables, and frequency for qualitative variables. Multivariate logistic regression models were fitted to assess the association between treatment-related variables (current treatment, interrupted treatment, previous treatment, and treatment pattern) with clinical, life style, and sociodemographic characteristics, controlled for possible confounding factors and interactions. The analyses were conducted using Stata (version MP $12^{\circledR}$ ) and R Project (version 3.1.2 ${ }^{\odot}$ ) to provide a $95 \%$ Confidence Interval and $p$-value $\leq 0.05$.

As a descriptive study, this research was not designed to assess any specific statistical hypothesis. Thus, the number of individuals included in the sample was not based on a statistical sample size calculation, but on practical considerations, such as viability of reference center.

\section{RESULTS}

A total of 564 patients with HCV genotype 3 were included in the sample, and their clinical and sociodemographic characteristics are presented in Table 1. The proportions of male and female patients were similar $(49.8 \%$ and $50.2 \%$, respectively) and mean age of the sample was 59.3 years $(S D=10.5)$. Most patients were classified as Caucasian/White (92.9\%).

Mean time since diagnosis was 103.0 months $(\mathrm{SD}=38.9$; 8.58 years $)$, with mean Body Mass Index (BMI) of $28.0(\mathrm{SD}=5.5)$. Alcohol and drug consumption were identified in $23 \%$ and $10.1 \%$ of patients, respectively.

Regarding disease severity, 318 (56.4\%) patients presented cirrhosis, with mean MELD score of 12.4 $(S D=6.3)$. Most patients $(64 \%)$ were classified as Child-Turcotte-Pugh Score level A. The most common extra-hepatic manifestations and comorbidities were systemic arterial hypertension (36.6\%), diabetes mellitus $(30 \%)$, hepatocellular carcinoma $(20.9 \%)$, liver transplantation $(13.5 \%)$, obesity $(12.4 \%)$ and depression (11.9\%).

Information on treatment pattern is shown in Table 2. Among HCV genotype 3 patients with information on treatment pattern, $25.2 \%(\mathrm{~N}=142)$ were treatment-naïve, and the most common reason for not treating was absence of fibrosis / mild fibrosis (22.5\%), followed by decompensated cirrhosis / hepatocellular carcinoma / waiting for transplantation (21.83\%) (Figure 1). 
Table 1: Clinical and sociodemographic characteristics of HCV genotype 3 patients.

\begin{tabular}{|c|c|c|}
\hline Characteristics & $\mathbf{N}$ & $\%$ \\
\hline \multicolumn{3}{|l|}{ Age } \\
\hline $18-29$ years & 3 & 0.5 \\
\hline $30-39$ years & 15 & 2.7 \\
\hline $40-49$ years & 73 & 12.9 \\
\hline $50-59$ years & 183 & 32.5 \\
\hline $60-69$ years & 211 & 37.4 \\
\hline$\geq 70$ years & 79 & 14 \\
\hline [Mean / SD] & 59.3 & 10.5 \\
\hline \multicolumn{3}{|l|}{ Gender } \\
\hline Female & 283 & 50.2 \\
\hline Male & 281 & 49.8 \\
\hline \multicolumn{3}{|l|}{ Race } \\
\hline Caucasian/White & 519 & 92 \\
\hline Black & 24 & 4.3 \\
\hline Brown & 20 & 3.5 \\
\hline Indigenous & 1 & 0.2 \\
\hline \multicolumn{3}{|l|}{ Anthropometric data } \\
\hline Height $(\mathrm{cm}-\mathrm{N}=155)[$ Mean $/ \mathrm{SD}]$ & 73.8 & 16.7 \\
\hline Weight $(\mathrm{Kg}-\mathrm{N}=$ 203) [Mean / SD] & 164.1 & 10.5 \\
\hline BMI (N = 152) [Mean / SD] & 28 & 5.5 \\
\hline \multicolumn{3}{|l|}{ Diagnosis } \\
\hline Time since diagnosis (months) [Mean / SD] & 103 & 38.9 \\
\hline \multicolumn{3}{|l|}{ Lifestyle behaviors } \\
\hline Alcohol consumption & 130 & 23 \\
\hline Other drug consumption & 57 & 10.1 \\
\hline \multicolumn{3}{|l|}{ Disease severity } \\
\hline Cirrhosis $^{1}$ & 318 & 56.4 \\
\hline MELD Score ( $N=219)$ [Mean / SD] & 12.4 & 6.3 \\
\hline Child-Turcotte-Pugh Score & 311 & 55.1 \\
\hline A & 199 & 64 \\
\hline B & 77 & 24.8 \\
\hline $\mathrm{C}$ & 35 & 11.2 \\
\hline \multicolumn{3}{|l|}{ Extra-hepatic manifestations and comorbidities ${ }^{2}$} \\
\hline Systemic Arterial Hypertension & 205 & 36.3 \\
\hline Diabetes Mellitus & 169 & 30 \\
\hline Hepatocellular carcinoma & 118 & 20.9 \\
\hline Liver Transplantation & 76 & 13.5 \\
\hline Obesity & 70 & 12.4 \\
\hline Depression & 67 & 11.9 \\
\hline Absence of extra-hepatic manifestations or comorbidities & 150 & 26.6 \\
\hline
\end{tabular}

Absence of extra-hepatic manifestations or comorbidities

${ }^{1}$ According to medical chart information or fibrosis evaluation.

${ }^{2}$ Only extra-hepatic manifestations and comorbidities with frequency $\geq 10 \%$ were showed. Other conditions reported were: thyroiditis, chronic kidney disease, cardiovascular disease, non-alcoholic steatohepatitis, dyslipidemia, arthralgia, glomerulopathy, biliary lithiasis, gastrointestinal cancer, cryoglobulinemia, hemochromatosis, biliary stenosis, vasculitis, lymphoma, porphyria cutanea tarda, autoimmune hepatitis, sjogren's syndrome and lichen planus.

BMI: Body Mass Index; MELD: Model for End-Stage Liver Disease 


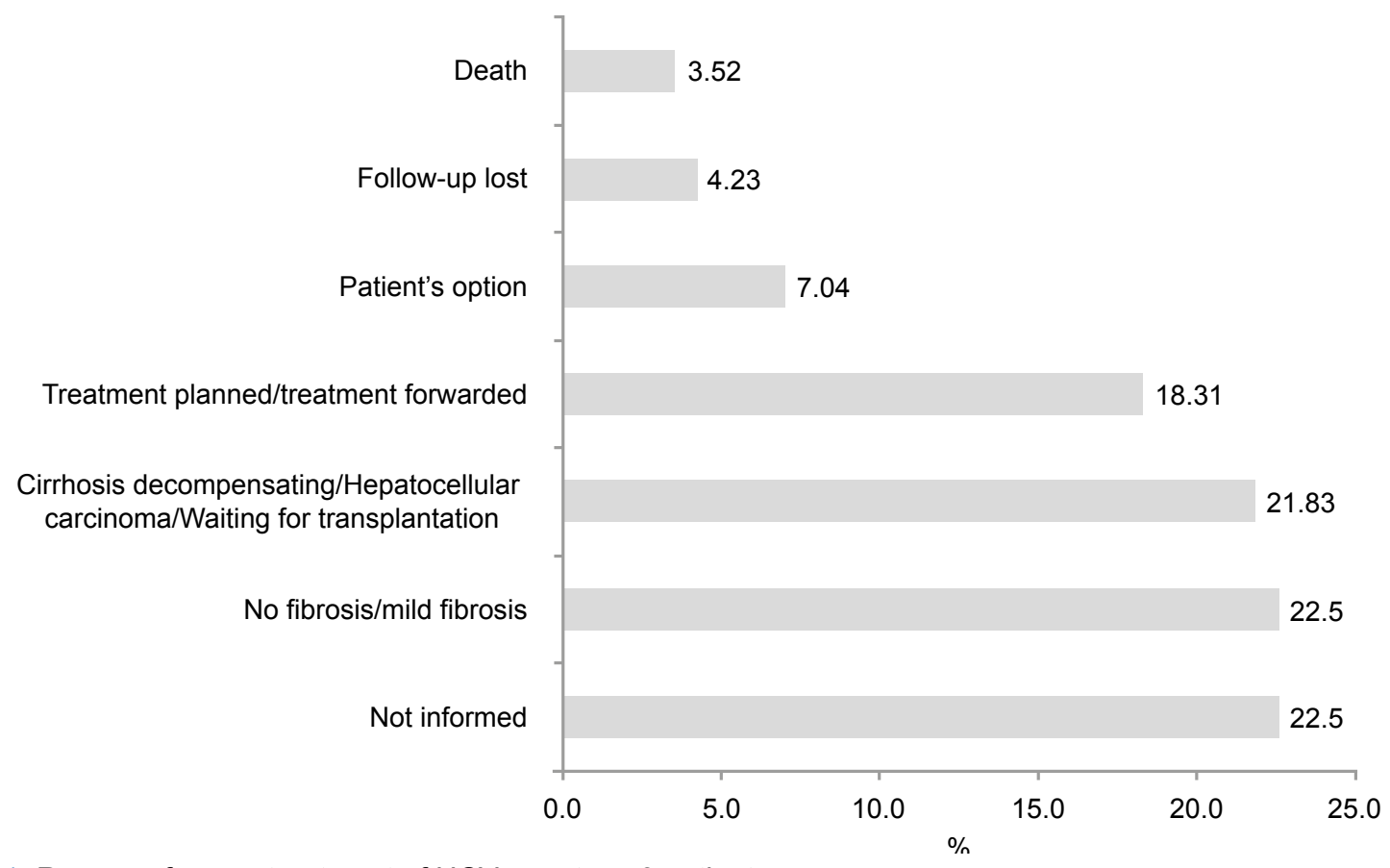

Figure 1: Reasons for non-treatment of HCV genotype 3 patients.

Table 2: Treatment pattern of HCV genotype 3 patients.

\begin{tabular}{lcc}
\hline Characteristics & $\mathbf{N}$ & $\%$ \\
\hline Non-treated patients & 142 & 25.2 \\
Treated patients & 421 & 74.8 \\
Patients currently treated & 49 & 11.6 \\
Patients without current treatment & 366 & 87 \\
Not informed & 6 & 1,4 \\
Number of treatments (N = 421) [Mean / SD] & 1.5 & 0.7 \\
\hline Patients currently treated (N = 49) & & \\
\hline Current treatment pattern & 43 & 87.8 \\
Sofosbuvir + Daclatasvir ( \pm Ribavirin) & 3 & 6.1 \\
Pegylated interferon \pm Ribavirin & 3 & 6.1 \\
Sofosbuvir + Pegylated interferon + Ribavirin & 16.6 & \\
Current treatment duration (weeks-N = 44) [Mean / SD] & & \\
\hline Patients without current treatment (N = 366) & & \\
\hline Last treatment pattern & 254 & 69.4 \\
Pegylated interferon \pm Ribavirin & 92 & 25.1 \\
Sofosbuvir + Daclatasvir ( \pm Ribavirin) & 16 & 4.4 \\
Sofosbuvir + Pegylated interferon + Ribavirin & 4 & 1.1 \\
Not informed & 27.2 & 15.5 \\
Last treatment duration (weeks-N = 313) [Mean / SD] & & \\
\hline Previous treatment characteristics (N = 388) & 235 \\
Sustained virologic response (N = 351) & 73 & 19.8 \\
Treatment interruption (N = 369) & & \\
Reasons for treatment interruption & 31 & 42.5 \\
Unspecified adverse event & 14 & 19.2 \\
Cirrhosis decompensating/hospitalization & 12 & 16.4 \\
Hematological adverse event & 9 & 12.3 \\
Poor adherence/follow-up lost & 3 & 4.1 \\
Death during treatment & 3 & 4.1 \\
Drug intolerance & 1 & 1.4 \\
Psychiatric adverse event & 1 &
\end{tabular}

* Variables described by frequency (\%). 
Among the patients treated $(\mathrm{N}=421 ; 74.8 \%$ of total sample), $11.6 \%(\mathrm{~N}=49 / 421)$ were receiving treatment during the study period, while $87 \%(\mathrm{~N}=366$ / 421) had received previous treatment, but were not under ongoing treatment. On average, patients received 1.5 treatments $(S D=0.7)$. Considering the patients currently treated $(\mathrm{N}=49)$, most of them were on sofosbuvir + daclatasvir ( \pm ribavirin) $(87.8 \%)$. The mean current treatment duration was 16.6 weeks $(S D=5.9)$.

Among patients who were not receiving treatment during the study, but had previous treatment, the mean therapy duration was 27.2 weeks $(S D=15.5)$, and the most common therapeutic regimen was pegylated interferon \pm ribavirin $(69.4 \%)$, followed by sofosbuvir + daclatasvir ( \pm ribavirin) $(25.1 \%)$.

Independently of current treatment status, 388 patients had at least one previous treatment. Of these, $67 \%$ achieved sustained virologic response in the last treatment, but treatment was interrupted in $19.8 \%$ of cases. Most frequent reasons for treatment interruption were: unspecified adverse events $(42.5 \%)$, decompensated cirrhosis/hospitalization (19.2\%), and hematologic adverse events (16.4\%).

Logistic regression models showed that patients were more likely to receive treatment if they were Caucasian/white $(O R=3.809$; $p$-value $=0.002)$, nonobese (OR $=2.595 ; p$-value $=0.007)$, transplanted $(\mathrm{OR}=5.010 ; p$-value $=0.001)$, presented longer time since diagnosis $(O R=1.010 ; p$-value $=0.004)$, and had cirrhosis according to fibrosis evaluation comparatively to those without fibrosis $(\mathrm{OR}=0.258$; $p$-value $=0.001)$ or mild fibrosis $(O R=0.480$; $\mathrm{p}$-value $=0.022)$. Patients with hepatocellular carcinoma had $64.1 \%(\mathrm{OR}=0.359 ; \mathrm{p}$-value $=0.036)$ less chance to be on current treatment during the study period than those without this condition; while patients with chronic kidney disease were 2.913-fold ( $p$-value $=0.025)$ more likely to have a treatment interruption than those without this disease.

Finally, models showed that patients presenting higher chance of receiving pegylated interferon \pm ribavirin than sofosbuvir + daclatasvir ( \pm ribavirin) in their last treatment were those who consumed alcohol $(O R=2.719 ; p$-value $=0.006)$ and those with non-alcoholic steatohepatitis $(\mathrm{OR}=6.067$; p-value $=0.044)$. Transplanted patients were $82.6 \%$ $(\mathrm{OR}=0.174 ; \mathrm{p}$-value $=<0.001)$ less likely to receive pegylated interferon \pm ribavirin than sofosbuvir + daclatasvir ( \pm ribavirin). Also, a one-month increase in time since diagnosis resulted in an increase of $0.7 \%(O R=1.007 ; p$-value $=0.032)$ in the chance of receiving pegylated interferon \pm ribavirin and not sofosbuvir + daclatasvir ( \pm ribavirin) in the last treatment. When compared to patients with cirrhosis (according to FibroScan), individuals with mild and severe fibrosis were 2.659 -fold $(p$-value $=0.012$ ) more likely and $62.4 \%(O R=0.376 ; p$-value $=0.013)$ less likely, respectively, of being treated with pegylated interferon \pm ribavirin compared to sofosbuvir + daclatasvir ( \pm ribavirin).

\section{DISCUSSION}

This study aimed to describe a large sample of patients with HCV genotype 3 who attended a specialized center in the South Region of Brazil, specifically addressing variables concerning treatment patterns and their contributing factors. Our dataset was able to comprehensively describe the clinical and demographic features of this subgroup of HCV patients, as well as how they are currently treated and which variables may contribute to explain the choice of treatment within the available therapeutic arsenal.

Gender distribution in the present study was balanced among HCV genotype 3 patients, similarly to a study conducted in China ${ }^{11}$. However, this distribution differs from studies conducted in Brazil ${ }^{12}$ and Pakistan ${ }^{13}$. These studies presented a higher proportion of male patients, which can be explained by the higher susceptibility of men to known risk factors ${ }^{12-14}$. Less than a quarter of patients in the sample (23\%) had records of alcohol consumption. This lifestyle behavior may play a role in the progression of HCV, although the mechanism is not totally elucidated. Also, alcohol is a relevant prognostic factor for HCV and for the development of cirrhosis ${ }^{15-17}$.

The presence of comorbidities/extra-hepatic manifestations negatively impacts the treatment and outcomes of HCV ${ }^{18,19}$. The most frequent coexistence conditions among the HCV genotype 3 $\mathrm{HCV}$ patients included in this sample were systemic arterial hypertension (36.3\%) and diabetes mellitus $(30 \%)$. These proportions were higher than those reported by Melo et al. ${ }^{12}$ in a population from São Paulo (systemic arterial hypertension-19\%; diabetes mellitus-11\%). This difference between the studies can be justified by the sample composition, since the referred study excluded patients who were coinfected, transplanted, alcohol-dependent and with end-stage renal disease ${ }^{12}$. The available literature indicates hypertension as the sixth most frequent HCV comorbidity with a known association with infection progression. In addition, if severe, hypertension is a relevant contraindication for the treatment of $\mathrm{HCV}^{19}$. Previous studies also reported that HCV patients have a higher risk of developing diabetes mellitus than healthy individuals or patients with other hepatic infections ${ }^{20,21}$, which may justify the high proportion of this comorbidity in our sample.

The presence of coexisting conditions showed a great influence on treatment characteristics. Obese 
patients were less likely to receive treatment compared to non-obese patients; and transplanted patients were more likely to receive treatment. The presence of hepatocellular carcinoma was associated with a lower chance of receiving treatment during the study, while chronic kidney disease was associated with treatment interruption. In fact, it is known that the presence of coexisting conditions can change the course of treatment, impacting on the effectiveness of the drugs ${ }^{18}$. Thus, health care providers must be aware of these conditions and consider them during the prescription decision process.

Currently, the first line for the treatment of HCV genotype $3 \mathrm{HCV}$ is the scheme consisting of sofosbuvir + daclatasvir \pm ribavirin, followed by the sofosbuvir + pegylated interferon + ribavirin scheme. In agreement with the guidelines of the Brazilian Ministry of Health $(\mathrm{MoH})$, these therapeutic regimens were the most frequent ongoing treatments in our sample $(87.8 \%$ and $6.1 \%$, respectively $)^{2}$. It is important to highlight that $6.1 \%$ of patients were being treated with pegylated interferon \pm ribavirin, a strategy recommended by the 2011 protocol version for genotypes 2 and $3^{10}$.

The most common last treatment received was pegylated interferon \pm ribavirin $(69.4 \%)$, which is consistent with the $2011 \mathrm{MoH}$ guidelines ${ }^{10}$. The sofosbuvir + daclatasvir \pm ribavirin regimen was the last treatment used by $25.1 \%$ of patients, which may be justified by the most recent introduction of this option in the $\mathrm{MoH} 2015$ guidelines ${ }^{8}$. Accordingly, the logistic regression model, when the time since diagnostic increased by one month, there was a $0.7 \%$ increase in the chance that the patient received pegylated interferon \pm ribavirin as last treatment, instead of sofosbuvir + daclatasvir \pm ribavirin.

Patients with mild or no fibrosis had less chance of receiving treatment than those with cirrhosis. Also, the most common reasons for not treating the infection were the absence of fibrosis / mild fibrosis $(22.5 \%)$ and decompensated cirrhosis / hepatocellular carcinoma / waiting for transplantation (21.8\%). These justifications for non-treatment are in accordance with the 2015 and 2017 protocols, which recommended treatment for severe fibrosis and cirrhosis only 8,9 . However, with the 2018 updated version, all stages of hepatic fibrosis, including patients with hepatocellular carcinoma, should receive treatment ${ }^{2}$.

The main goals of HCV treatment are to achieve sustained virologic response in 12 or 24 weeks, according to the therapy administered ${ }^{2,9}$. The HCV genotype plays an important role in sustained virologic response and previous studies reported that genotype 3 had higher rates of sustained virologic response than genotypes 1 and $4^{22}$. In our sample, most patients obtained sustained virologic response after their last treatment $(67 \%)$. This proportion is within the $66-80 \%$ range reported by other authors ${ }^{22}$.

According to most recent HCV protocol, the definition of the severity of infection is fundamental for therapy decision making ${ }^{2}$. More than half of this sample had cirrhosis, signalizing severe disease. In contrast, Fernandes et al. ${ }^{23}$ found a $7 \%$ frequency of cirrhosis in other Brazilian study ${ }^{23}$. However, these authors did not specify the genotype of the sample, which may explain the difference, since previous studies have reported an association between genotype 3 and more advanced stages of fibrosis, cirrhosis, and hepatocellular carcinoma ${ }^{6,12,22}$.

The present study was conducted in the state of Rio Grande do Sul and covers exclusively patients with HCV genotype 3. Since the distribution of HCV genotypes varies among Brazilian regions and even between states in the same region ${ }^{5}$, the generalization of data must be done with caution. Another potential limitation of this study may be related to missing data or under notification, since it was conducted with a retrospective review of medical records. However, this limitation is applicable to all participants and is unlikely to result in a systematic bias.

There are few studies exclusively about HCV genotype 3 in Brazil. Thus, the present research provides an important contribution in describing a large sample of patients with this genotype. Treatment characteristics were influenced by the presence of comorbidities and complications of HCV, such as obesity, hepatic transplantation, hepatocellular carcinoma, and chronic kidney disease.

The most prescribed treatment today was sofosbuvir + daclatasvir \pm ribavirin and is in accordance with current $\mathrm{HCV} \mathrm{MoH}$ guidelines. On the other hand, there is still a small group of patients treated with pegylated interferon \pm ribavirin, a treatment schedule considered suboptimal. The proportion of patients who achieved a sustained virologic response with last treatment was similar to other studies conducted with genotype 3 patients. Besides, the subgroup of patients with no/mild fibrosis who were not receiving treatment may face a change in treatment patterns in the near future, since they are now considered eligible for treatment according to a more recent version of the national HCV protocol.

\section{Conflict of Interests}

The authors have nothing to disclose.

\section{Funding}

This research was funded by Abbvie biopharmaceutical company. 


\section{REFERENCES}

1. World Health Organization. Global Hepatitis Report, 2017. Geneva: WHO Press; 2017.

2. Brasil. Ministério da Saúde. Secretaria de Vigilância em Saúde. Departamento de DST Aids e Hepatites Virais. Protocolo clínico e diretrizes terapêuticas para hepatite $c$ e coinfecções. Brasília, DF; 2018.

3. Simmonds $\mathrm{P}$, Bukh J, Combet C, Deléage $\mathrm{G}$, Enomoto N, Feinstone $S$, et al. Consensus proposals for a unified system of nomenclature of hepatitis $C$ virus genotypes. Hepatology. 2005;42(4):962-73.

4. Blach S, Zeuzem S, Manns M, Altraif I, Duberg A-S, Muljono DH, et al. Global prevalence and genotype distribution of hepatitis $C$ virus infection in 2015: a modelling study. Lancet Gastroenterol Hepatol. 2017;2(3):161-76.

5. Campiotto S, Pinho JRR, Carrilho FJ, Silva LC, Souto FJD, Spinelli V, et al. Geographic distribution of hepatitis C virus genotypes in Brazil. Braz J Med Biol Res. 2005;38(1):41-9

6. Kanwal F, Kramer JR, llyas J, Duan $Z$, El-Serag HB. HCV genotype 3 is associated with an increased risk of cirrhosis and hepatocellular cancer in a national sample of U.S. Veterans with HCV. Hepatology. 2014;60(1):98-105.

7. Buti $M$, Esteban R. Hepatitis $C$ virus genotype 3: a genotype that is not 'easy-to-treat'. Expert Rev Gastroenterol Hepatol. 2015;9(3):375-85.

8. Brasil. Ministério da Saúde. Secretaria de Vigilância em Saúde. Departamento de DST Aids e
Hepatites Virais. Protocolo clínico e diretrizes terapêuticas para Hepatite $C$ e coinfecções. Brasília, DF; 2015.

9. Brasil. Ministério da Saúde. Secretaria de Vigilância em Saúde. Departamento de DST Aids e Hepatites Virais. Protocolo clínico e diretrizes terapêuticas para Hepatite $C$ e coinfecções. Brasília, DF; 2017.

10. Brasil. Ministério da Saúde. Secretaria de Vigilância em Saúde. Departamento de DST Aids e Hepatites Virais. Protocolo clínico e diretrizes terapêuticas para Hepatite $C$ e coinfecções. Brasília, DF; 2011.

11. Niu Z, Zhang P, Tong Y. Age and gender distribution of Hepatitis $C$ virus prevalence and genotypes of individuals of physical examination in WuHan, Central China. Springerplus. 2016;5(1):1557.

12. Melo IC, Ferraz MLG, Perez RM, Emori CT, Uehara SNO, CarvalhoFilho RJ, et al. Do differences exist between chronic hepatitis $C$ genotypes 2 and 3 ? Rev Soc Bras Med Trop. 2014;47(2):143-8.

13. Ayaz H, Mahmood H, Nazir $\mathrm{H}$, Sikandar M, Bhatti A, Naseer A. Distribution of Genotype 3 in Hepatitis C Patients \& its Association with Gender and Age. J Rawalpindi Med Coll Stud Supp. 2016;20(S-2):141-3.

14. Morais MTM, Oliveira TJ. Perfil epidemiológico e sociodemográfico de portadores de Hepatite $\mathrm{C}$ de um município do Sudoeste Baiano. Rev Saúde.com. 2015;11(2):137-46.

15. Younossi ZM, Zheng L, Stepanova M, Venkatesan C, Mir HM. Moderate, excessive or heavy alcohol consumption: each is significantly associated with increased mortality in patients with chronic hepatitis C. Aliment Pharmacol Ther. 2013;37(7):703-9.

16. Peters MG, Terrault NA. Alcohol use and hepatitis C. Hepatology. 2002;36(501):s220-5.

17. Ostapowicz G, Watson KJ, Locarnini SA, Desmond P V. Role of alcohol in the progression of liver disease caused by hepatitis $C$ virus infection. Hepatology. 1998;27(6):1730-5.

18. El-Zayadi AR. Hepatitis C comorbidities affecting the course and response to therapy. World $J$ Gastroenterol. 2009;15:4993-9.

19. Louie KS, St Laurent S, Forssen UM, Mundy LM, Pimenta JM. The high comorbidity burden of the hepatitis $C$ virus infected population in the United States. BMC Infect Dis. 2012;12:86.

20. Hammerstad SS, Grock SF, Lee HJ, Hasham A, Sundaram N, Tomer Y. Diabetes and Hepatitis C: a twoway association. Front Endocrinol (Lausanne). 2015;6:134.

21. White DL, Ratziu V, El-Serag HB. Hepatitis $C$ infection and risk of diabetes: a systematic review and meta-analysis. $J$ Hepatol. 2008;49(5):831-44.

22. Chan A, Patel K, Naggie S. Genotype 3 infection: the last stand of Hepatitis C virus. Drugs. 2017;77(2):131-44.

23. Fernandes FF, Ferraz ML, Andrade LE, Dellavance A, Terra C, Pereira $\mathrm{G}$, et al. Enhanced liver fibrosis panel as a predictor of liver fibrosis in chronic Hepatitis C patients. J Clin Gastroenterol. 2015;49(3):235-41. 